\title{
Application of Geographic Information Technology in Shared Cycling
}

\author{
Xiaoxuan Wang ${ }^{1, a, *}$, Jing Chen ${ }^{2}$ \\ ${ }^{1}$ Huali College Guangdong University of Technology, Hebei, 511325, China \\ ${ }^{2}$ Huali College Guangdong University of Technology, Shandong, 511325, China \\ a rser@foxmail.com \\ *corresponding author
}

Keywords: Geographic Information Technology, Cycling, Combined Use, Current Situation.

\begin{abstract}
With the continuous improvement of living standards, people's awareness of environmental protection is growing day by day, shared bicycle also appeared in the streets of the city. Sharing bicycle refers to the enterprise in campus, subway station, bus stop, residential area, business district, public service area and so on to provide bicycle sharing service, which is a new type of green sharing economy. Sharing bicycle is essentially a new type of transportation leasing business-bike leasing business, which mainly relies on the carrier for bicycle. People use mobile GIS remote sensing information technology to find parking and changing places, which greatly promotes the use of shared bikes.
\end{abstract}

\section{Introduction}

We all know that geographic information technology plays a very important role in people's daily life. The use of this technology strengthens the connection between product companies and users by strengthening the software data we use in our daily life. Geographic information technology strengthens the interrelation between people, changes people's way of life, and breaks the traditional way of thinking. Geographic information systems, sometimes referred to as "geoinformation systems" or "resource and environmental information systems ". It is a specific very important spatial information system. It is a technical system for the collection, storage, management, operation, analysis, display and description of the geographic distribution data in the whole or part of the Earth's surface space, supported by computer hardware and software systems. Combining this technology with shared bikes can better apply shared bikes to our lives.

\section{The Connotation of Geographic Information Technology}

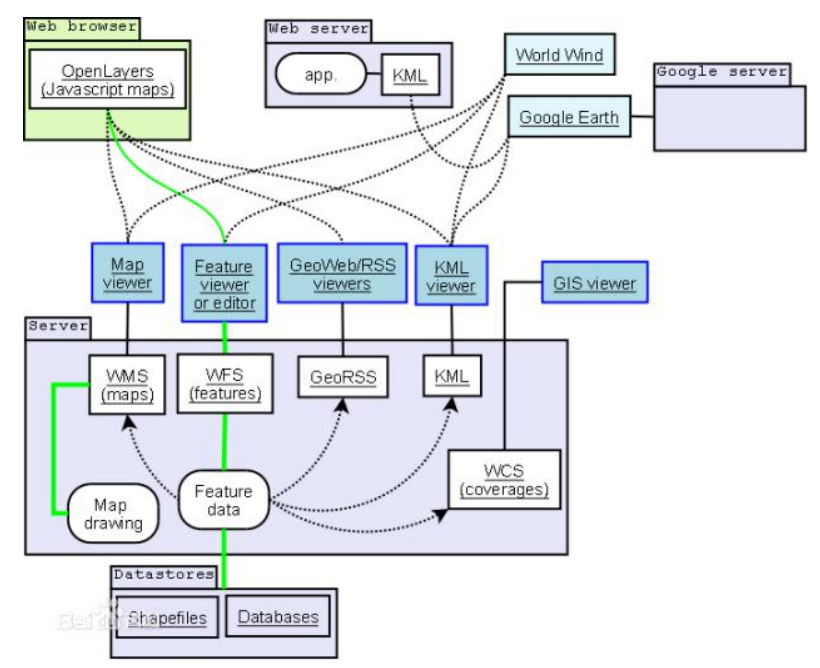

Figure 1 Data

GIS processes and manages a variety of geospatial entity data and their relationships, including 
spatial positioning data, graphic data, remote sensing Image data, attribute data, etc., are used to analyze and deal with various phenomena and processes distributed in a certain geographical area to solve complex planning, decision-making and management problems. Remote sensing is the use of remote sensors from the air to detect the properties of ground objects, according to the principle of different objects to the spectrum of different responses to the principle, identify all kinds of ground objects, with the meaning of distant perception of things. That is, the use of remote sensors on aircraft, spacecraft, satellites and other flying objects above the ground to collect ground data and to obtain information from them to identify features by recording, transmitting, analysing and interpreting them (see figure 1).

\section{The Development of Shared Cycling}

With the development of shared bicycle industry, there are all kinds of shared bicycle in our street. The emergence of shared bicycle not only effectively solves our travel problems, alleviates the traffic congestion in the city, but also can adapt to the green environmental protection policy of the city and alleviate the pollution situation of the city. People pay for the two-dimensional code on shared bikes, but because of the increasing number of shared bikes, there are also some problems in the storage of bicycles, many people do not have public awareness, random parking of bicycles, resulting in difficult maintenance, placement, shared bicycle workers are very inconvenient to find, and even people destroy the bicycle positioning system, for our use, the development of shared bicycle is worrying[1].

\section{Use of Geographic Information Technology on Shared Cycling}

Through the continuous improvement of science and technology, the use of geographic information technology in shared bicycle can effectively alleviate these conditions and help the better development of shared bicycle. A large number of shared bicycle and shared tram need geographic information technology to better control and management. As we all know, the use of bicycles greatly facilitates our daily travel and alleviates traffic congestion in cities. Sharing bikes operates based on Internet technology, big data and cloud computing, geographic information technology, and GPS positioning. The two-dimensional code unlocking technology is also a derivative of Internet mode, which makes the development of shared bikes dependent on geographic information technology, which makes it more clear that there is no sharing bicycle without geographic information technology[2](Fig.2).

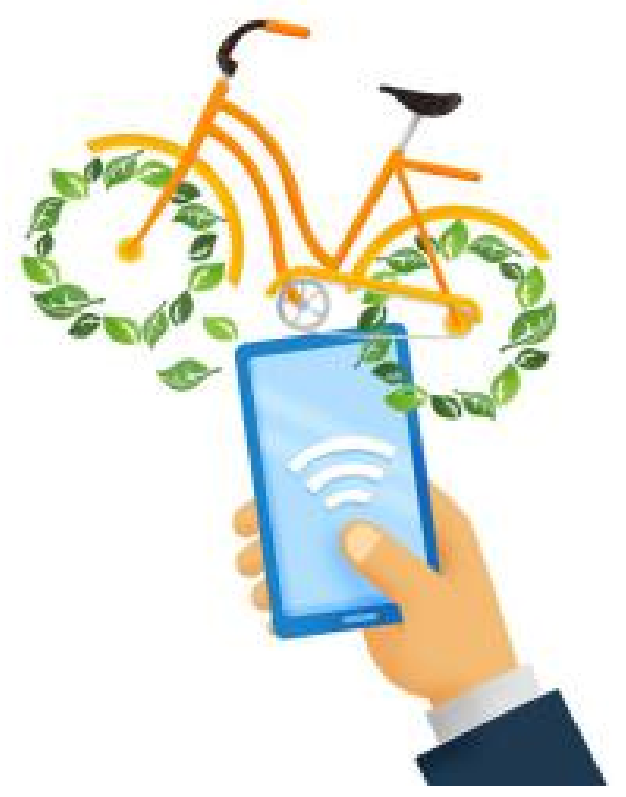

Figure 2 Sharing bikes 


\subsection{Cycling Client}

Shared bicycle client is a kind of mobile phone app application software, users download the application software, and input their personal information to obtain the system permission, and finally can get the right to use the bicycle, and users can use the query function in the software system to obtain the parking place of the bicycle, but also can monitor the bicycle, can avoid the vehicle theft and can better collect the corresponding data, so as to improve the user experience to achieve better results.

\subsection{Remote Sensing Information Technology}

Remote sensing data also plays an important role in data collection and is composed of multiple sensors attached to the platform. Sensors include cameras, digital scanners and lidar, while platforms are usually made up of aircraft and satellites. Most of the digital data comes from picture interpretation and aerial photographs. soft copy workstations are used to digitize features obtained directly from stereo image pairs of digital images. These systems allow data to be captured in two or three dimensions, and their elevation is directly derived from stereogram measurements based on the principle of photographic measurements. Nowadays, analog aerial photos are scanned and then entered into a soft copy system, but this step can be omitted as high-quality digital cameras become cheaper. Satellite remote sensing provides another important source of spatial data, where satellites use different sensor packages to passively measure the reflection coefficients of parts of electromagnetic spectrum or radio waves emitted from active sensors such as radar, and remote sensing collection can be further processed to identify objects of interest and classes such as landcovered raster data.

Mobile remote sensing information technology is a mosaic system application, which is equipped with a large number of content memory, mosaic system can use GIS remote sensing technology to carry out satellite navigation and positioning system, according to the information analysis application to personalized setting. We all know that the emergence of shared bikes is convenient for us to travel, but we usually encounter a situation where bikes are everywhere when we don't need to share bikes, but when we need to share bikes when we don't, we become anxious and restless. When remote sensing GIS technology is applied to bicycle software, we can search through our mobile phones to find the number of bikes around us and the location of the bikes.[3].

There are two types of shared bikes, one with free parking, no location restrictions, the other with an electronic card to rent and a designated location when returning (figure 3), which not only exacerbates people's travel problems but also takes up a lot of time. The application of geographic information technology has helped people to solve this problem, convenient for people to travel, but also convenient for the management of bicycles.

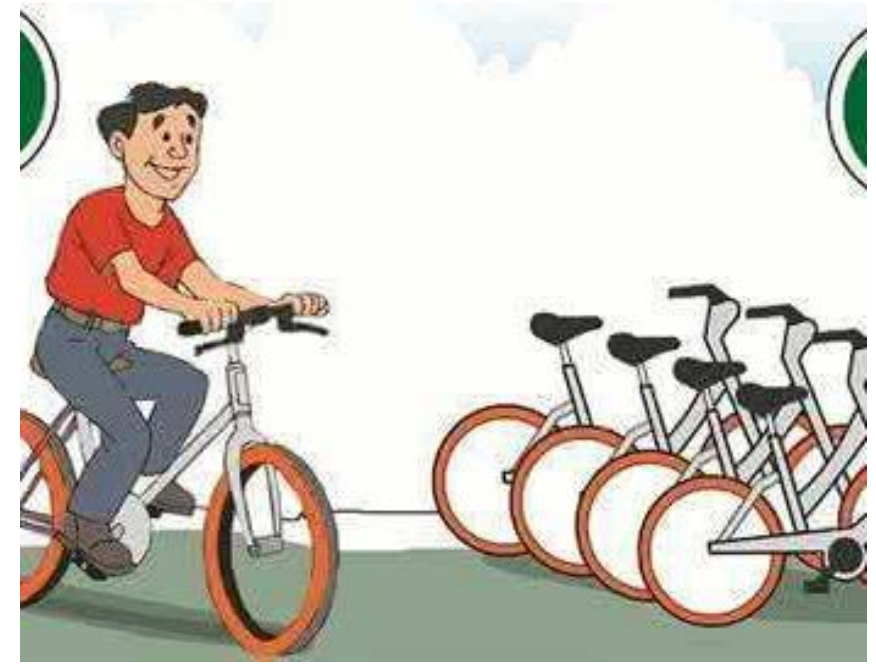

Figure 3 Designated locations 


\section{Advice on the Management of Cycling in GIS}

Application of Geographic Information Technology in Shared Cycling Convenient Use of Cycling Conducting inquiries and integration and Conduct its data Analysis and processing, we all know that shared bicycle is concentrated on the road, using geographic information technology system to monitor the bicycle, linear geographical features are particularly obvious, we can fully use GIS remote sensing information technology to manage shared bicycle, so that shared bicycle has a stronger visualization and visualization features.

The government urban management department should strengthen the management of sharing bicycles, establish and improve the management system, fully apply the geographic information technology, combine with the actual situation of regional management, better designate the development mechanism to help the construction of the city more perfect. Carrying out the scale management of the area management and the reasonable control of the quantity of shared bicycle can realize the scale control of the delivery area, avoid the road crisis caused by the excessive delivery, and affect the beauty of the city. To speed up the construction of bicycle parking points in light of the actual situation, the government departments should design the key roads according to the number of bicycles to be put in and the people's riding conditions. At the same time, they should do a good job of publicity, protect people's lives and safety, and consider the actual needs of the residents. Through the monitoring and management of vehicles through geographic information technology, the real implementation of vehicle fixed-point parking, bicycle delivery companies should cooperate with government departments to establish a corresponding shared bicycle platform, through geographic information technology better service users. Full implementation of service management functions, real-time monitoring, targeted, and the use of satellite positioning and other technical means to set up electronic fence area[4].

\section{Conclusion}

Through the use of geographic information technology in shared bicycle, we can understand that to help users or a better sense of use, but also to help the customer terminal platform to collect user information, better improve the product structure, but also to help government departments to monitor the status of bicycle real-time, the implementation of government functions. The application of GIS technology will be more extensive, and the interaction of shared bicycle and GIS technology makes this business model more popular. Therefore, while strengthening the sharing of bicycles, strengthen supervision, open a new era of interactive mode, this will be very worth looking forward to. Many disciplines benefit from GIS technology, and the active GIS market has led to low cost and continuous improvement of hardware and software for GIS components. these developments have in turn led to the wider application of this technology in science, government, business, and industry, with applications including real estate, public health, crime maps, defence, sustainable development, natural resources, landscape architecture, archaeology, community planning, transportation, and logistics.

\section{Acknowledgements}

This research has been financed by The Young Innovative Talents Project of General Colleges and Universities in Guangdong Province” The Cooperative Application Model of BIM and GIS in Urban Construction”(2016KQNCX211)

\section{References}

[1] Shi, Dichao., Shi, Xiaoming., Fang, Fang. Application of Geographic Information Technology in Shared Cycling. Geospatial Information, vol. 16, no. 01, pp. 7-8+55-56, 2018.

[2] Chen, Pengxu. Application of Internet of things technology in shared bicycle. Internal combustion engine and accessories, 2018. 
[3] Xu, Yanhua., Bao, Yingying., Geng, Miao. Application of Internet of Things in Urban Shared Cycling. Digital Technology and Applications, vol. .36, no. 336(06), pp. 213-214, 2018.

[4] Peng, Xiuping., Li, Zhongwen. Analysis of Internet of things behind shared bicycle. Information and Computer, no. 18, 2017. 